\title{
The transition to turbulence in slowly diverging subsonic submerged jets
}

\author{
P. S. Landa ${ }^{1}$ and P. V. E. McClintock ${ }^{2}$ \\ ${ }^{1}$ Physics Department, Lomonosov Moscow State University, Moscow 119899 Russia \\ ${ }^{2}$ Department of Physics, Lancaster University, Lancaster LA1 4YB, United Kingdom
}

(Received 2 August 2011; accepted 9 February 2012; published online 14 March 2012)

\begin{abstract}
We address the problem of how turbulence is created in a submerged plane jet, near to the nozzle from which it issues. We do so by making use of a WKB-like asymptotic expansion for approximate solution of a complex, linear, fourth-order differential equation describing small deviations from the steady-state stream function. The result is used as a generating solution for application of the asymptotic Krylov-Bogolyubov method, enabling us to find the spatial and temporal spectra of the turbulence in the first approximation. We have thus been able to find the complex eigenvalues and eigenfunctions, i.e., the natural waves. We show that, for any given set of parameters, there is a continuum of frequencies and, for each frequency, a continuum of phase velocities. Correspondingly, there is an infinite number of wavelengths. It follows that there is no unique dispersion law and, because of perturbations (however, small they may be), a regular temporal spectrum does not exist even in cases where the spatial spectrum is regular. (C) 2012 American Institute of Physics. [http://dx.doi.org/10.1063/1.3693141]
\end{abstract}

\section{INTRODUCTION}

Turbulence is widespread in nature and technology, and forms part of common experience. Formally, it may be defined as a far-from-equilibrium state of a nonlinear physical system with many degrees of freedom, and it exists in many different variants. Of these, vortex turbulence ${ }^{1,2}$ is probably the best known. A common feature of turbulent systems lies in energy transfer between the scale or frequency at which energy from an external source is pumped into the system and the different scale or frequency at which it can be transformed to heat via dissipative processes. Wave turbulence has been seen or postulated, e.g., among phonons in solids, ${ }^{3}$ in optical fibers and nonlinear optical media, ${ }^{4}$ on vibrating plates ${ }^{5-7}$ and the surfaces of ferrofluids (liquids that become strongly magnetized in a magnetic field) ${ }^{8,9}$ for sound waves in oceanic waveguides, ${ }^{10}$ as magnetic turbulence in interstellar gases, ${ }^{11}$ shock waves in the solar wind coupled to the earth's magnetosphere, ${ }^{12}$ surface waves on liquid hydrogen, ${ }^{13}$ and second sound in superfluid helium. ${ }^{14,15}$ Frequently, turbulence involves a cascade-like transfer of turbulent energy towards the high frequency domain, ${ }^{14,16}$ which is where dissipation mainly occurs, but inverse cascades where the energy flows towards larger length scales (lower frequencies) can sometimes occur too. ${ }^{15,17}$ Although turbulence has been studied for many years, and in many different contexts, the nature of the transition to turbulence has remained something of an enigma.

We are not aware of any single, clear, universally accepted definition of turbulence. The reason is that turbulence can exist in different circumstances: in systems with an absolute instability, e.g., in closed systems or in certain biological ones, turbulence is excited via feedback and is a self-oscillatory process; in open systems with large coefficients of amplification for disturbances, turbulence results from an amplification of external or internal noise. ${ }^{18}$ In what follows, we define turbulence as a state in which there are random vortex waves in a distributed system whose characteristics either do not depend at all on the initial conditions, or depend only weakly on them. ${ }^{19}$

In this paper, we consider a particular, well-characterised, example of the transition to turbulence: in the jet flow that occurs when a fluid is injected fast through a nozzle into stationary fluid of the 
same kind. Such flows quickly become turbulent, and how the turbulence arises is a very challenging problem both from the scientific and practical points of view. It was solved theoretically in its very simplest approximation (a jet flow from a point source), by Landau ${ }^{20}$ but real jets are of course considerably more complicated. Submerged jets form the main element of the open-jet wind tunnels used for structural tests of aircrafts, ships, and rockets and there are many other important applications. To control the level of turbulence in the jet, an understanding of its internal turbulent processes is needed.

Because of the convective character of the instability, turbulence in submerged jets is not a self-oscillatory process, as initially suggested by Gorelik and Landau (see e.g., Rytov's reminiscences discussed in Ref.19) but, rather, it should be viewed as arising via a nonequilibrium phase transition. ${ }^{19,21-24}$ This insight allowed us to propose effective methods for the control of turbulence, different from those used for self-oscillatory systems like e.g., impinging jets ${ }^{25}$ where feedback can cause the self-excitation of oscillations. One such method is the application of acoustic forcing to the jet.

We have concluded ${ }^{17}$ that the turbulence arises through strong amplification of the weak random disturbances that are always present in jets and, especially, within the nozzle exit section. Within the jet, at distances close to the nozzle, the turbulent pulsations are small, and for their calculation we can use a quasi-linear theory, e.g., the Krylov-Bogolyubov asymptotic method for distributed systems. ${ }^{26}$ For any given harmonic constituent of the stream function, we find a linear equation containing an unknown complex wave number. It was shown that, for large Reynolds numbers, ordinary methods of solving even the linearized three-dimensional Navier-Stokes equations for a slowly-diverging plane flow yield random results due to instability. In the present work, we have therefore used a WKB-like asymptotic expansion method for the approximate solution of a complex, linear, fourthorder differential equation describing small deviations from the steady-state stream function. This equation contains a large parameter proportional to the root-mean-square of the Reynolds number. The method allows us to find the complex eigenvalues and eigenfunctions. The real part of the eigenvalue describes the real wave number of a hydrodynamical wave of the frequency in question, and the imaginary part defines its spatial growth rate. It is the latter that determines the stability of the jet flow. We will show below that, for any value of the wave frequency, there are an infinite number of eigenvalues of the wave number and eigenfunctions. This circumstance arises on account of the inhomogeneity of the system in the region of the boundary layer.

We note that in earlier linear theories of jet instability, ${ }^{27-31}$ the problem was solved by use of the Euler equations for an inviscid fluid. This approach was consistent with the widespread belief that jet flows may be calculated in the approximation of inviscid fluid (assertions to this effect appear in many papers and books). The authors of these works succeeded in finding approximate eigenfunctions and eigenvalues from the condition that any bounded solution of the initial equations must vanish far from the jet axis (but did not compare their results with experiment). In Ref. 17, we used the same approach, but starting from the Navier-Stokes equations for the case a plane jet. For any given set of parameters this leads to a single eigenvalue for a given frequency and distance from the nozzle. Critically, however, we overlooked the fact that the eigenvalues depend on the point where the condition for the vanishing solution is applied. We note en passant the large body of work based on rapid distortion theory (RDT), ${ }^{32,33}$ which was introduced for the calculation of how developed turbulence is distorted when it passes rapidly through a region of large-scale straining motions, and later extended to analyse inhomogeneous turbulent flows, including some effects of boundaries.

In the present paper, we employ a different approach to treat the transition to turbulence. For each set of parameters, we solve the Navier-Stokes equations with initial conditions specified on the jet axis and for boundary conditions specified far from the axis, beyond the boundary layer. We sew these solutions together at a point inside the boundary layer. We will show that, in doing so, the eigenvalues depend on the sewing point, i.e., for any value of the frequency, we find a continuous set of eigenvalues - complex wave numbers-and corresponding eigenfunctions. Their occurrence is similar to a phenomenon that occurs in an inhomogeneous optical medium where the propagation of a monochromatic wave of a given frequency results in a continuous set of waves propagating at different phase velocities. 
The authors of Refs. 27, 28,30,31 refer to their approach as a WKB-method, because the jet diverges slowly in comparison with the variation of jet velocity within any cross-section. When we refer to a WKB-like method in the present work, however, we mean something rather different: a WKB-like technique for approximating the solution of the linear non-self-adjoint boundary-value problem. We emphasize that, in what follows below, all conditions for the applicability of the WKBmethod are fulfilled at every point (with the exception of the matching points themselves, which are of zero dimension).

The approach we introduce falls into three main parts:

1. Splitting of the variables into regular and random constituents. Our procedure differ from the standard one used by the most theoretical physicists, in which they split the solutions of the initial equations into mean values and the deviations from these means. Instead, we split the solutions into regular (stationary) and random contributions. For many problems (e.g, for nonlinear optics) the two procedures coincide; but for hydrodynamical problems, because of the quadratic nonlinearity, the mean values depend on the deviations from them, leading to a well-known problem of closure of the equations. Our procedure allows us to avoid this difficulty. The penalty we pay is that the equations for the random constituents may become more complicated than those in the conventional procedure. Our approach is restricted to small turbulence levels.

2. Numerical solution of the linearized equations for the random constituents of the stream function with initial conditions, both at the jet axis, and with boundary conditions far from the axis, outside the boundary layer.

3. Sewing together of the resultant solutions and calculation of the eigenvalues of the wavenumbers.

In Sec. II, we introduce the main equations needed for the description of a plane jet flow, and then split them into equations describing its regular and random components in Secs. II A and II B respectively. In Sec. III, we show how the Krylov-Bogolyubov method can be used to solve the equations for the random constituents of the flow, thus describing how turbulence develops close to the nozzle. The physical implications of the results obtained are discussed in Sec. IV. In Sec. V, we summarize and draw conclusions.

\section{THE MAIN EQUATIONS FOR A PLANE JET}

For simplicity, we consider a plane jet issuing from a nozzle of width $2 d$ (Fig. 1). Neglecting compressibility, we may describe the processes occurring in such a jet by the two-dimensional Navier-Stokes equation for the stream function $\Psi(t, x, y) \cdot{ }^{20} \mathrm{It}$ is related to the longitudinal $(U)$ and transverse $(V)$ components of the flow velocity by $U(t, x, y)=\partial \Psi / \partial y, V(t, x, y)=-\partial \Psi / \partial x$. In dimensionless coordinates $x^{\prime}=x / d, y^{\prime}=y / d$ and time $t^{\prime}=U_{0} t / d$, where $U_{0}$ is the mean flow velocity in the nozzle center, this equation may be written as

$$
\frac{\partial \Omega^{\prime}}{\partial t^{\prime}}-\frac{\partial \Psi^{\prime}}{\partial x^{\prime}} \frac{\partial \Omega^{\prime}}{\partial y^{\prime}}+\frac{\partial \Psi^{\prime}}{\partial y^{\prime}} \frac{\partial \Omega^{\prime}}{\partial x^{\prime}}-\frac{2}{\operatorname{Re}} \Delta^{\prime} \Omega^{\prime}=0
$$

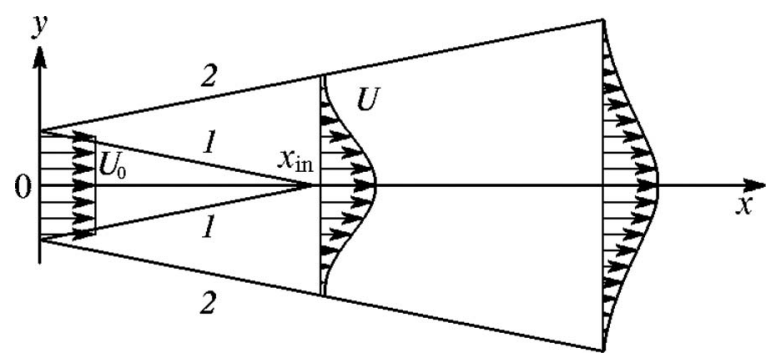

FIG. 1. Schematic image of a diverging free jet illustrating the change of its mean velocity profile and widening of the mixing layer. Curves 1 and 2 correspond, respectively, to the internal and external boundaries of the mixing layer. 
where $\Delta^{\prime}$ is the Laplacian in terms of $x^{\prime}$ and $y^{\prime}, \operatorname{Re}=2 U_{0} d / v$ is the Reynolds number, $v$ is kinematic viscosity,

$$
\Omega^{\prime}\left(t^{\prime}, x^{\prime}, y^{\prime}\right)=\Delta^{\prime} \Psi^{\prime}\left(t^{\prime}, x^{\prime}, y^{\prime}\right)
$$

is the vorticity. From this point onwards the primes will be dropped.

It should be noted that, corresponding to the dimensionless time, the circular frequencies $\omega=2 \pi f$ are measured in units of $\mathrm{S}=\omega d / U_{0} \equiv \pi \mathrm{St}$, where $\mathrm{St}=2 f d / U_{0}$ is the Strouhal number.

The authors of all classical works on turbulence (e.g., Refs. 20 and 34), starting from Reynolds, split the solution of the initial nonlinear equations into mean values and random disturbances. Because of the quadratic nonlinearity in the hydrodynamical equations the mean values depend on the deviations from them. Consequently, there is a problem of closure for these equations. To avoid it, we split the solution of Eqs. (1) and (2) into dynamical (regular) and random constituents. ${ }^{17}$ It is clear that such approach is reasonable only where the level of turbulence is small. We now consider in turn the equations for the dynamical and random parts, in Secs. II A and II B, respectively.

\section{A. The equations for dynamical constituents}

In autonomous jets the dynamical constituents are described by stationary Navier-Stokes equations

$$
\begin{gathered}
\Omega_{\mathrm{d}}(x, y)=\frac{\partial U_{\mathrm{d}}(x, y)}{\partial y}-\frac{\partial V_{\mathrm{d}}(x, y)}{\partial x}, \\
\frac{\partial U_{\mathrm{d}}(x, y)}{\partial x}+\frac{\partial V_{\mathrm{d}}(x, y)}{\partial y}=0, \\
U_{\mathrm{d}}(x, y) \frac{\partial \Omega_{\mathrm{d}}(x, y)}{\partial x}+V_{\mathrm{d}}(x, y) \frac{\partial \Omega_{\mathrm{d}}(x, y)}{\partial y}-\frac{2}{\operatorname{Re}}\left(\frac{\partial^{2} \Omega_{\mathrm{d}}(x, y)}{\partial x^{2}}+\frac{\partial^{2} \Omega_{\mathrm{d}}(x, y)}{\partial y^{2}}\right)=0,
\end{gathered}
$$

whereas the random constituents are described by the equations for deviations from the stationary solutions. The deviations are caused mainly by the sources of disturbance at the nozzle output section.

Because Eqs. (3)-(5) cannot be solved analytically, and even their numerical solution presents insurmountable difficulties, we set the profile of longitudinal velocity component to be of the form

$$
U_{\mathrm{d}}(x, y)=\frac{1}{1+\tanh \left(q / \delta_{00}+r_{0}\right)}\left[1-\tanh \left(q \frac{|y|-1}{\delta_{0}(x)}-r(x)\right)\right],
$$

where $r(x)$ and $\delta_{0}(x)$ are unknown functions of $x, \delta_{0}(x) \equiv \delta_{1}(x)+\delta_{2}(x)$ is the boundary layer thickness which is assumed to be equal to $\delta_{00}$ for $x=0, \delta_{1}(x)$ and $\delta_{2}(x)$ are the thicknesses of internal and external parts of the boundary layer, and $r(x)=r_{0}$ for $x=0$. This approach is similar to the well known Galerkin method. ${ }^{35}$ To calculate the unknown functions, we have used the conservation laws for the fluxes of momentum and energy. Usually these laws are derived from Reynolds equations for the mean values of these fluxes, ${ }^{36,37}$ and that is why they contain the so-called turbulent viscosity. In Ref. 17, these laws were derived approximately from Eqs. (3)-(5).

By applying the conservation laws we have found

$$
r(x) \approx r_{0}=0.5, \quad \delta_{0}(x)=\sqrt{\delta_{00}^{2}+\frac{32 q^{2}}{3 \operatorname{Re}} x}, \quad \delta_{1}(x) \approx \frac{\delta_{0}(x)}{3}, \delta_{2}(x) \approx \frac{2 \delta_{0}(x)}{3} .
$$

The value of $\delta_{00}=\delta_{0}(0)$ depends at the conditions of the nozzle outflow. In the case of laminar flow, when the boundary layer may be approximately described by the Blasius function, ${ }^{20,38} \delta_{00}$ is inversely proportional to $\sqrt{\mathrm{Re}}$. We also set $\delta_{00}=1 /\left(b_{0} \sqrt{\mathrm{Re}}\right)$, where $b_{0}=0.1$. 
The expressions for $V_{\mathrm{d}}(x, y)$ and $\Omega_{\mathrm{d}}(x, y)$ can be found by exact solution of Eqs. (3) and (4). As a result, we obtain

$$
\begin{aligned}
& V_{\mathrm{d}}(x, y)=-\frac{16 q \operatorname{sign} y}{3 \operatorname{Re} \delta_{0}(x)\left(1+\tanh \left(q / \delta_{00}+r_{0}\right)\right)}\left[\frac{q(|y|-1)}{\delta_{0}(x)} \tanh \left(\frac{q(|y|-1)}{\delta_{0}(x)}-r_{0}\right)\right. \\
& \left.-\frac{q}{\delta_{0}(x)} \tanh \left(\frac{q}{\delta_{0}(x)}+r_{0}\right)-\ln \frac{\cosh \left(q(|y|-1) / \delta_{0}(x)-r_{0}\right)}{\cosh \left(q / \delta_{0}(x)+r_{0}\right)}\right], \\
& \Omega_{\mathrm{d}}(x, y)=-\frac{q \operatorname{sign} y}{\delta_{0}(x)\left(1+\tanh \left(q / \delta_{00}+r_{0}\right)\right)}\left\{\left(1+\frac{256 q^{4}(|y|-1)^{2}}{9 \delta_{0}^{4}(x) \operatorname{Re}^{2}}\right) \cosh ^{-2}\left(\frac{q(|y|-1)}{\delta_{0}(x)}-r_{0}\right)\right. \\
& -\frac{256 q^{2}}{9 \delta_{0}^{2}(x) \operatorname{Re}^{2}}\left[\frac{q^{2}}{\delta_{0}^{2}(x)} \cosh ^{-2}\left(\frac{q}{\delta_{0}(x)}+r_{0}\right)-\frac{q}{\delta_{0}(x)}\left((|y|-1) \tanh \left(\frac{q(|y|-1)}{\delta_{0}(x)}-r_{0}\right)\right.\right. \\
& \left.\left.\left.-\tanh \left(\frac{q}{\delta_{0}(x)}+r_{0}\right)\right)+\ln \frac{\cosh \left(q(|y|-1) / \delta_{0}(x)-r_{0}\right)}{\cosh \left(q / \delta_{0}(x)+r_{0}\right)}\right]\right\} .
\end{aligned}
$$

Taking into account that $q / \delta_{0}(x) \gg 1$, we can find approximate expressions for $V_{\mathrm{d}}$ and $\Omega_{\mathrm{d}}$ that are valid at large values of $|y|$

$$
V_{\mathrm{d}}(x, \pm \infty) \approx \mp \frac{16 q r_{0}}{3 \delta_{0}(x) \operatorname{Re}}, \quad \Omega_{\mathrm{d}}(x, \pm \infty) \approx \mp \frac{256 q^{3} r_{0}}{9 \delta_{0}^{3}(x) \mathrm{Re}^{2}} .
$$

Figure 2 shows plots of $U_{\mathrm{d}}(x, y), V_{\mathrm{d}}(x, y)$, and $\Omega_{\mathrm{d}}(x, y)$ versus $y$ for $b_{0}=0.1, q=3, r_{0}=0.5$, $\mathrm{Re}=25000, x=0$, and $x=8$. For all values of $y$ except for narrow intervals near $y= \pm 1$, we see that the quantities $U_{\mathrm{d}}(x, y), V_{\mathrm{d}}(x, y)$, and $\Omega_{\mathrm{d}}(x, y)$ are nearly constant. The constant transverse velocity constituent for $|y|>1$ directed towards the jet axis describes the entrainment of ambient fluid with the jet flow.

It should be emphasized that the results obtained here relate only to the dynamical constituents of the velocity and vorticity. Random constituents have a strong influence upon the thickness of the boundary layer, its dependence on the distance from the nozzle, and values of the mean velocities and mean vorticity.

\section{B. The equations for random constituents}

Substituting

$$
\begin{aligned}
& U(t, x, y)=U_{\mathrm{d}}(x, y)+\frac{\partial \Psi_{\mathrm{r}}(t, x, y)}{\partial y}, \quad V(t, x, y)=V_{\mathrm{d}}(x, y)-\frac{\partial \Psi_{\mathrm{r}}(t, x, y)}{\partial x}, \\
& \Omega(t, x, y)=\Omega_{\mathrm{d}}(x, y)+\Omega_{\mathrm{r}}(t, x, y)
\end{aligned}
$$

into initial equations, we obtain for random constituents $\Psi_{\mathrm{r}}(t, x, y)$ and $\Omega_{\mathrm{r}}(t, x, y)$ the following equations:

$$
\begin{aligned}
& \Omega_{\mathrm{r}}-\Delta \Psi_{\mathrm{r}}=0, \\
& \frac{\partial \Omega_{\mathrm{r}}}{\partial t}+U_{\mathrm{d}}(x, y) \frac{\partial \Omega_{\mathrm{r}}}{\partial x}+V_{\mathrm{d}}(x, y) \frac{\partial \Omega_{\mathrm{r}}}{\partial y}-\Omega_{\mathrm{d} y}(x, y) \frac{\partial \Psi_{\mathrm{r}}}{\partial x}+\Omega_{\mathrm{d} x}(x, y) \frac{\partial \Psi_{\mathrm{r}}}{\partial y}-\frac{2}{\operatorname{Re}} \Delta \Omega_{\mathrm{r}} \\
& =\frac{\partial \Psi_{\mathrm{r}}}{\partial x} \frac{\partial \Omega_{\mathrm{r}}}{\partial y}-\frac{\partial \Psi_{\mathrm{r}}}{\partial y} \frac{\partial \Omega_{\mathrm{r}}}{\partial x},
\end{aligned}
$$

where $\Omega_{\mathrm{d} x}(x, y)=\partial \Omega_{\mathrm{d}}(x, y) / \partial x, \quad \Omega_{\mathrm{d} y}(x, y)=\partial \Omega_{\mathrm{d}}(x, y) / \partial y$. 

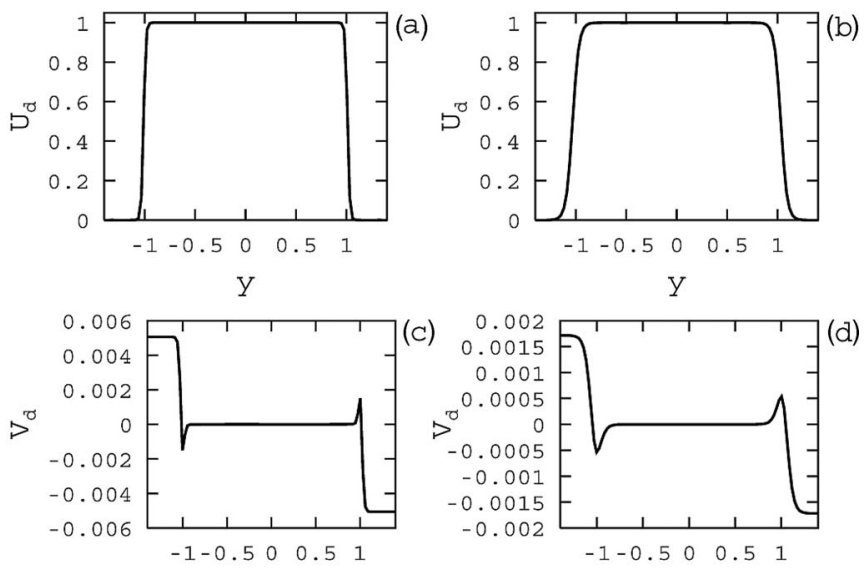

$\mathrm{Y}$

Y
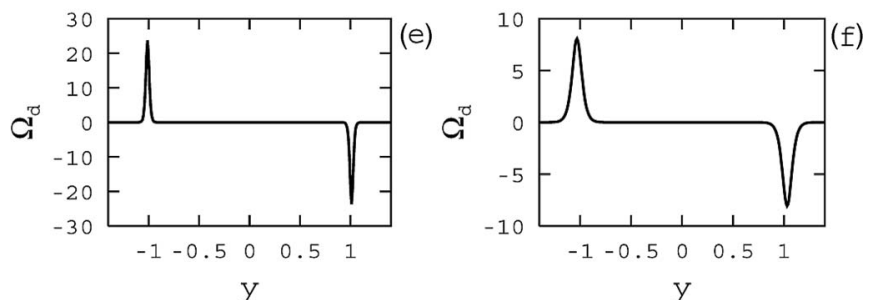

FIG. 2. Plots of $U_{\mathrm{d}}(x, y), V_{\mathrm{d}}(x, y)$, and $\Omega_{\mathrm{d}}(x, y)$ versus $y$ for $b_{0}=0.1, q=3, r_{0}=0.5$, $\operatorname{Re}=25000$ : (a) $U_{\mathrm{d}}(0, y)$; (b) $U_{\mathrm{d}}(8$, $y) ;\left(\right.$ c) $V_{\mathrm{d}}(0, y) ;\left(\right.$ d) $V_{\mathrm{d}}(8, y) ;\left(\right.$ e) $\Omega_{\mathrm{d}}(0, y)$; and (f) $\Omega_{\mathrm{d}}(8, y)$.

\section{USING THE KRYLOV-BOGOLYUBOV METHOD FOR STUDYING THE DEVELOPMENT OF TURBULENCE NOT FAR FROM THE JET NOZZLE}

To describe the development of turbulence, we can assume that the right-hand side of Eq. (12) is of the order of a conditional small parameter $\epsilon$. In this case, Eqs. (11) and (12) can be solved approximately by a method similar to the Krylov-Bogolyubov method for spatially extended systems. ${ }^{17,26}$ We therefore seek a solution in the form of a series in $\epsilon$

$$
\begin{aligned}
& \Omega_{\mathrm{r}}(t, x, y)=\Omega_{0}(t, x, y)+\epsilon r_{1}(t, x, y)+\epsilon^{2} r_{2}(t, x, y)+\ldots, \\
& \Psi_{\mathrm{r}}(t, x, y)=\psi_{0}(t, x, y)+\epsilon s_{1}(t, x, y)+\epsilon^{2} s_{2}(t, x, y)+\ldots, \\
& u(t, x, y)=\frac{\partial \Psi_{\mathrm{r}}(t, x, y)}{\partial y}=u_{0}(t, x, y)+\epsilon u_{1}(t, x, y)+\epsilon^{2} u_{2}(t, x, y)+\ldots,
\end{aligned}
$$

where $\Omega_{0}(t, x, y)$ and $\psi_{0}(t, x, y)$ are generative solutions of Eqs. (11) and (12), $r_{1}(t, x, y), r_{2}(t, x, y)$, $\ldots, s_{1}(t, x, y), s_{2}(t, x, y), \ldots$ are unknown functions, and $u_{0}(t, x, y)=\partial \psi_{0}(t, x, y) / \partial y, u_{1}(t, x, y)$ $=\partial s_{1}(t, x, y) / \partial y, u_{2}(t, x, y)=\partial s_{2}(t, x, y) / \partial y, \ldots$

It should be emphasized that because of the quadratic nonlinearity the contribution of nonlinear terms into turbulent processes can be estimated only by using higher approximations of the KrylovBogolyubov method, beginning from the first. In this work, we will restrict our calculations only to linear approximation.

\section{A. Generative equations (linear approximation)}

Setting the right-hand side of Eq. (12) to zero, and taking account of Eq. (11), we obtain the following generative equation for the random constituent of the stream function:

$$
\frac{\partial \Delta \psi_{0}}{\partial t}+U_{\mathrm{d}}(x, y) \frac{\partial \Delta \psi_{0}}{\partial x}+V_{\mathrm{d}}(x, y) \frac{\partial \Delta \psi_{0}}{\partial y}-\Omega_{\mathrm{d} y}(x, y) \frac{\partial \psi_{0}}{\partial x}+\Omega_{\mathrm{d} x}(x, y) \frac{\partial \psi_{0}}{\partial y}-\frac{2}{\operatorname{Re}} \Delta \Delta \psi_{0}=0 .
$$


We seek a particular solution for Eq. (14) in the form of a sum of running waves with Strouhal number $\mathrm{S}$, amplitude $f(\mathrm{~S}, x, y)$, and a slowly varying complex wave number $Q(\mathrm{~S}, x)$

$$
\psi_{0}(t, x, y)=\frac{1}{2 \pi} \int_{-\infty}^{\infty} f(\mathrm{~S}, x, y) \exp \left[i\left(\mathrm{~S} t-\int_{0}^{x} Q(\mathrm{~S}, x) d x\right)\right] d \mathrm{~S} .
$$

The complex wave numbers $Q(\mathrm{~S}, x)$ may be represented as $Q(\mathrm{~S}, x)=\mathrm{S} / v_{\mathrm{ph}}(\mathrm{S}, x)+i \Gamma(\mathrm{S}, x)$, where $v_{\mathrm{ph}}$ is the wave phase velocity and $\Gamma$ is the spatial growth rate (gain factor).

Taking into account that the jet diverges slowly, we can represent the function $f(\mathrm{~S}, x, y)$ and the wave number $Q(\mathrm{~S}, x)$ as series in another conditional small parameter $\mu \sim 1 / \sqrt{\mathrm{Re}}$ characterizing the slowness of the jet divergence

$$
f(\mathrm{~S}, x, y)=f_{0}(\mathrm{~S}, x, y)+\mu f_{1}(\mathrm{~S}, x, y)+\ldots, \quad Q(\mathrm{~S}, x)=Q_{0}(\mathrm{~S}, x)+\mu Q_{1}(\mathrm{~S}, x)+\ldots,
$$

where $f_{0}(\mathrm{~S}, x, y), f_{1}(\mathrm{~S}, x, y), \ldots$ are unknown functions vanishing, along with their derivatives, at $y= \pm \infty$.

Substituting Eq. (15) into Eq. (14) and retaining only the first term in the expansion (16), we obtain the following equation for $f_{0}(\mathrm{~S}, x, y)$ :

$$
L_{0}\left(Q_{0}(\mathrm{~S}, x)\right) f_{0}(\mathrm{~S}, x, y)=0,
$$

where

$$
\begin{aligned}
& L_{0}\left(Q_{0}(\mathrm{~S}, x)\right)=i\left(\mathrm{~S}-U_{\mathrm{d}}(x, y) Q_{0}(\mathrm{~S}, x)\right)\left(\frac{\partial^{2}}{\partial y^{2}}-Q_{0}^{2}(\mathrm{~S}, x)\right)+V_{\mathrm{d}}(x, y)\left(\frac{\partial^{3}}{\partial y^{3}}-Q_{0}^{2}(\mathrm{~S}, x) \frac{\partial}{\partial y}\right) \\
& +i Q_{0}(\mathrm{~S}, x) \Omega_{\mathrm{d} y}(x, y)+\Omega_{\mathrm{d} x}(x, y) \frac{\partial}{\partial y}-\frac{2}{\operatorname{Re}}\left(\frac{\partial^{4}}{\partial y^{4}}-2 Q_{0}^{2}(\mathrm{~S}, x) \frac{\partial^{2}}{\partial y^{2}}+Q_{0}^{4}(\mathrm{~S}, x)\right) .
\end{aligned}
$$

Equation (17), in view of Eq. (18), together with the boundary conditions requiring function $f_{0}$ and its derivatives to be vanishing at $y= \pm \infty$, describes a non-self-adjoint boundary-value problem, whose solution allows us to find complex eigenvalues of $Q_{0}(\mathrm{~S}, x)$ and corresponding eigenfunctions.

Outside the boundary layer (for $y \geq y_{\infty}$ and $0 \leq y \leq y_{1}$ ), where $y_{\infty}$ is a sufficiently large value of $y$ and $y_{1}$ is a value of $y$ smaller than the inner boundary of the boundary layer, the functions $U_{\mathrm{d}}(x$, $y), V_{\mathrm{d}}(x, y)$, and $\Omega_{\mathrm{d} x}(x, y)$ are almost independent of $y$. Therefore in these regions of $y$ the solution of Eq. (17) can be found analytically. Taking account of the requirement that they vanish at large values of $y$, and assuming that $f_{0}(\mathrm{~S}, x, y)$ are odd functions of $y$, these solutions may be written as

$$
f_{0}(\mathrm{~S}, x, y)=\left\{\begin{array}{l}
C_{11}(x) \sinh \left(B_{11}(x) y\right)+C_{12}(x) \sinh \left(B_{12}(x) y\right) \text { for } 0 \leq y \leq y_{1}, \\
C_{21}(x) \exp \left(B_{21}(x) y\right)+C_{22}(x) \exp \left(B_{22}(x) y\right) \text { for } y \geq y_{\infty},
\end{array}\right.
$$

where

$$
B_{11}(x)=O_{0}(\mathrm{~S}, x), \quad B_{12}(x)=\sqrt{Q_{0}^{2}(\mathrm{~S}, x)+\frac{i\left(\mathrm{~S}-Q_{0}(\mathrm{~S}, x)\right) \mathrm{Re}}{2}}
$$

are roots of the characteristic equation in the region $0 \leq y \leq y_{1}$, and $B_{21}(x), B_{22}(x)$ are roots of the characteristic equation

$$
\begin{aligned}
& B^{4}-\frac{V_{\mathrm{d}}(x, \infty) \mathrm{Re}}{2} B^{3}-\left(2 Q_{0}^{2}+\frac{i \mathrm{SRe}}{2}\right) B^{2}-\frac{\operatorname{Re}}{2}\left(\frac{\partial \Omega_{\mathrm{d}}(x, \infty)}{\partial x}-Q_{0}^{2} V_{\mathrm{d}}(x, \infty)\right) \\
& \times B+Q_{0}^{4}+\frac{i \mathrm{~S} Q_{0}^{2} \operatorname{Re}}{2}=0
\end{aligned}
$$


in the region $y \geq y_{\infty}$ having negative real parts, $C_{11}(x), C_{12}(x), C_{21}(x)$, and $C_{22}(x)$ are arbitrary slow functions of $x$. It should be noted that $\left|B_{11}\right|$ and $\left|B_{21}\right|$ are of order 1 , whereas $\left|B_{12}\right|$ and $\left|B_{22}\right|$ are of order $\sqrt{R e}$.

It follows from here that, for any value of $y$, the general solution of Eq. (17) can be written as

$$
f_{0}(\mathrm{~S}, x, y)=\left\{\begin{array}{l}
C_{11}(x) f_{11}(\mathrm{~S}, x, y)+C_{12}(x) f_{12}(\mathrm{~S}, x, y) \text { for } 0 \leq y \leq y_{0}, \\
C_{21}(x) f_{21}(\mathrm{~S}, x, y)+C_{22}(x) f_{22}(\mathrm{~S}, x, y) \text { for } y \geq y_{0},
\end{array}\right.
$$

where $y_{0}$ is a point inside the boundary layer, and $f_{11}(\mathrm{~S}, x, y), f_{12}(\mathrm{~S}, x, y), f_{21}(\mathrm{~S}, x, y)$, and $f_{22}(\mathrm{~S}, x, y)$ are linearly independent solutions of Eq. (17) satisfying the following conditions:

$$
\begin{aligned}
& f_{11}(\mathrm{~S}, x, 0)=0,\left.\quad \frac{\partial f_{11}(\mathrm{~S}, x, y)}{\partial y}\right|_{y=0}=B_{11}(x),\left.\quad \frac{\partial^{2} f_{11}(\mathrm{~S}, x, y)}{\partial y^{2}}\right|_{y=0}=0, \\
& \left.\frac{\partial^{3} f_{11}(\mathrm{~S}, x, y)}{\partial y^{3}}\right|_{y=0}=B_{11}^{3}, \quad f_{12}(\mathrm{~S}, x, 0)=0,\left.\quad \frac{\partial f_{12}(\mathrm{~S}, x, y)}{\partial y}\right|_{y=0}=B_{12}(x), \\
& \left.\frac{\partial^{2} f_{12}(\mathrm{~S}, x, y)}{\partial y^{2}}\right|_{y=0}=0,\left.\quad \frac{\partial^{3} f_{12}(\mathrm{~S}, x, y)}{\partial y^{3}}\right|_{y=y_{\infty}}=B_{12}^{3}(x), \\
& \begin{array}{l}
f_{21}\left(\mathrm{~S}, x, y_{\infty}\right)=1,\left.\quad \frac{\partial f_{21}(\mathrm{~S}, x, y)}{\partial y}\right|_{y=y_{\infty}}=B_{21}(x),\left.\quad \frac{\partial^{2} f_{21}(\mathrm{~S}, x, y)}{\partial y^{2}}\right|_{y=y_{\infty}}=B_{21}^{2}(x), \\
\left.\frac{\partial^{3} f_{21}(\mathrm{~S}, x, y)}{\partial y^{3}}\right|_{y=y_{\infty}}=B_{21}^{3}(x), \quad f_{22}\left(\mathrm{~S}, x, y_{\infty}\right)=1,\left.\quad \frac{\partial f_{22}(\mathrm{~S}, x, y)}{\partial y}\right|_{y=y_{\infty}}=B_{22}(x), \\
\left.\frac{\partial^{2} f_{22}(\mathrm{~S}, x, y)}{\partial y^{2}}\right|_{y=y_{\infty}}=B_{22}^{2}(x),\left.\quad \frac{\partial^{3} f_{22}(\mathrm{~S}, x, y)}{\partial y^{3}}\right|_{y=y_{\infty}}=B_{22}^{3}(x) .
\end{array}
\end{aligned}
$$

Solutions (22) should be sewn together at some point $y=y_{0}$ inside the boundary layer. The sewing conditions are as follows:

$$
\begin{aligned}
& C_{11}(x) f_{11}\left(\mathrm{~S}, x, y_{0}\right)+C_{12}(x) f_{12}\left(\mathrm{~S}, x, y_{0}\right)=C_{21}(x) f_{21}\left(\mathrm{~S}, x, y_{0}\right)+C_{22}(x) f_{22}\left(\mathrm{~S}, x, y_{0}\right), \\
& \left.C_{11}(x) \frac{\partial f_{11}(\mathrm{~S}, x, y)}{\partial y}\right|_{y=y_{0}}+\left.C_{12}(x) \frac{\partial f_{12}(\mathrm{~S}, x, y)}{\partial y}\right|_{y=y_{0}}+C_{y=y_{0}}+\left.C_{22}(x) \frac{\partial f_{22}(\mathrm{~S}, x, y)}{\partial y}\right|_{y=y_{0}}, \\
& =\left.C_{21}(x) \frac{\partial f_{21}(\mathrm{~S}, x, y)}{\partial y}\right|_{y=y_{0}}+\left.C_{12}(x) \frac{\partial^{2} f_{12}(\mathrm{~S}, x, y)}{\partial y^{2}}\right|_{y=y_{0}} \\
& \left.C_{11}(x) \frac{\partial^{2} f_{11}(\mathrm{~S}, x, y)}{\partial y^{2}}\right|_{y=y_{0}}+\left.C_{22}(x) \frac{\partial^{2} f_{22}(\mathrm{~S}, x, y)}{\partial y^{2}}\right|_{y=y_{0}}, \\
& =\left.C_{21}(x) \frac{\partial^{2} f_{21}(\mathrm{~S}, x, y)}{\partial y^{2}}\right|_{y=y_{0}}+\left.\left.C_{12}(x) \frac{\partial^{3} f_{12}(\mathrm{~S}, x, y)}{\partial y^{3}}\right|_{y=y_{0}} \frac{\partial^{3} f_{11}(\mathrm{~S}, x, y)}{\partial y^{3}}\right|_{y=y_{0}}+\left.C_{22}(x) \frac{\partial^{3} f_{22}(\mathrm{~S}, x, y)}{\partial y^{3}}\right|_{y=y_{0}} .
\end{aligned}
$$

The eigenvalues of $Q_{0}(\mathrm{~S}, x)$ should be found from the condition for the vanishing of the determinant of the system of Eqs. (24). This determinant may be written as

$$
\begin{aligned}
& D\left(Q_{0}\left(\mathrm{~S}, x, y_{0}\right)\right) \\
& \quad=r_{23}\left(\mathrm{~S}, x, y_{0}\right) q_{01}\left(\mathrm{~S}, x, y_{0}\right)-r_{13}\left(\mathrm{~S}, x, y_{0}\right) q_{02}\left(\mathrm{~S}, x, y_{0}\right)+r_{12}\left(\mathrm{~S}, x, y_{0}\right) q_{03}\left(\mathrm{~S}, x, y_{0}\right) \\
& \quad+r_{03}\left(\mathrm{~S}, x, y_{0}\right) q_{12}\left(\mathrm{~S}, x, y_{0}\right)-r_{02}\left(\mathrm{~S}, x, y_{0}\right) q_{13}\left(\mathrm{~S}, x, y_{0}\right)+r_{01}\left(\mathrm{~S}, x, y_{0}\right) q_{23}\left(\mathrm{~S}, x, y_{0}\right),
\end{aligned}
$$


where

$$
\begin{aligned}
& q_{i j}\left(\mathrm{~S}, x, y_{0}\right)=\left.\left.\frac{\partial^{i} f_{11}(\mathrm{~S}, x, y)}{\partial y^{i}}\right|_{y=y_{0}} \frac{\partial^{j} f_{12}(\mathrm{~S}, x, y)}{\partial y^{j}}\right|_{y=y_{0}}-\left.\left.\frac{\partial^{j} f_{11}(\mathrm{~S}, x, y)}{\partial y^{j}}\right|_{y=y_{0}} \frac{\partial^{i} f_{12}(\mathrm{~S}, x, y)}{\partial y^{i}}\right|_{y=y_{0}}, \\
& r_{i j}\left(\mathrm{~S}, x, y_{0}\right)=\left.\left.\frac{\partial^{i} f_{21}(\mathrm{~S}, x, y)}{\partial y^{i}}\right|_{y=y_{0}} \frac{\partial^{j} f_{22}(\mathrm{~S}, x, y)}{\partial y^{j}}\right|_{y=y_{0}}-\left.\left.\frac{\partial^{j} f_{21}(\mathrm{~S}, x, y)}{\partial y^{j}}\right|_{y=y_{0}} \frac{\partial^{i} f_{22}(\mathrm{~S}, x, y)}{\partial y^{i}}\right|_{y=y_{0}} .
\end{aligned}
$$

A direct numerical calculation of $D\left(Q_{0}\left(\mathrm{~S}, x, y_{0}\right)\right.$, starting from initial equations, for large Reynolds numbers gives random values on account of the fact that $q_{i j}\left(\mathrm{~S}, x, y_{0}\right)$ and $r_{i j}\left(\mathrm{~S}, x, y_{0}\right)$ are differences between large numbers of the same order. One way to avoid these difficulties lies in the appropriate change of variables (see Ref. 17). Another way lies in the approximate solution of the initial equation by using a method similar to the WKB-method suggested by Wentsel, Kramers, and Brillouin in 1926 (Refs. 39-41) mainly for the problems of quantum mechanics. ${ }^{54,55}$ We note en passant that the WKB-method actually goes back to earlier works by Poincaré, Birkhoff, and Rayleigh ${ }^{42-44}$ and then it evolved further with Tamarkin. ${ }^{45,46}$ Later the WKB-method was set forth in Refs. 47-50): in Refs. 47 and 48 only for second order differential equations, and in Refs. 49 and 50 for higher order differential equations of a certain kind.

\section{Using the WKB-like method}

For using the WKB-like method, we take into account that $V_{\mathrm{d}}(x, y) / U_{\mathrm{d}}(x, y) \sim 1 / \lambda$, where $\lambda=\sqrt{R e}$ is a large parameter. It is therefore convenient to rewrite Eq. (17) in terms of $v_{\mathrm{d}}(x, y)$ $=V_{\mathrm{d}}(x, y) \lambda \sim U_{\mathrm{d}}(x, y)$ and to resolve it with respect to higher derivative. As a result, we find

$$
\begin{aligned}
& \frac{\partial^{4} f_{0}}{\partial y^{4}}-2 Q_{0}^{2} \frac{\partial^{2} f_{0}}{\partial y^{2}}+Q_{0}^{4} f_{0}-\frac{\lambda v_{\mathrm{d}}(x, y)}{2}\left(\frac{\partial^{3} f_{0}}{\partial y^{3}}-Q_{0}^{2} \frac{\partial f_{0}}{\partial y}\right) \\
- & \frac{\lambda^{2}}{2}\left[i\left(\mathrm{~S}-U_{\mathrm{d}}(x, y) Q_{0}\right)\left(\frac{\partial^{2} f_{0}}{\partial y^{2}}-Q_{0}^{2} f_{0}\right)+\Omega_{\mathrm{d} x}(x, y) \frac{\partial f_{0}}{\partial y}+i Q_{0} \Omega_{\mathrm{d} y}(x, y) f_{0}\right]=0 .
\end{aligned}
$$

According to the main idea of the WKB-method, a partial solution of Eq. (27) may be sought as

$$
f_{0}(x, y)=\exp \left(\lambda \int g(x, y) d y\right) \sum_{j=0}^{\infty} \varphi_{j}(x, y) \lambda^{-j},
$$

where $g(x, y)$ and $\varphi_{j}(x, y)$ are unknown functions. Substituting Eq. (28) into Eq. (27), restricting expansion (28) to the term of order $\lambda^{0}$ and equating the coefficients of $\lambda^{4}$, we obtain for $g(x, y)$ the following equation:

$$
g^{2}(x, y)\left(2 g^{2}(x, y)-v_{\mathrm{d}}(x, y) g(x, y)-i\left(\mathrm{~S}-U_{\mathrm{d}}(x, y) Q_{0}\right)\right)=0 .
$$

Equation (29) has four roots: two multiple zero roots and two different finite ones

$$
g_{1,2}(x, y)=\frac{v_{\mathrm{d}}(x, y) \mp \sqrt{v_{\mathrm{d}}^{2}(x, y)+8 i\left(\mathrm{~S}-U_{\mathrm{d}}(x, y) Q_{0}\right)}}{4} .
$$

It may be easily shown that only $g_{1}(x, y)$ has a negative real part for large values of $|y|$.

In the case of zero roots the coefficient of $\lambda^{3}$ vanishes. Equating the coefficients of $\lambda^{2}$, we find the equation for $\phi_{0}(x, y)$

$$
\begin{aligned}
& i\left(\mathrm{~S}-U_{\mathrm{d}}(x, y) Q_{0}\right)\left(\frac{\partial^{2} \phi_{0}(x, y)}{\partial y^{2}}-Q_{0}^{2} \phi_{0}(x, y)\right)+\Omega_{\mathrm{d} x}(x, y) \frac{\partial \phi_{0}(x, y)}{\partial y} \\
& +i Q_{0} \Omega_{\mathrm{d} y}(x, y) \phi_{0}(x, y)=0 .
\end{aligned}
$$

Equation (31) coincides with the corresponding Euler equation. 

of $y$

Outside the boundary layer Eq. (31) becomes an equation whose coefficients are independent

$$
\begin{aligned}
& \frac{\partial^{2} \phi_{0}(x, y)}{\partial y^{2}}-Q_{0}^{2}(x) \phi_{0}(x, y)=0 \quad\left(y \leq y_{1}\right), \\
& i \mathrm{~S}\left(\frac{\partial^{2} \phi_{0}(x, y)}{\partial y^{2}}-Q_{0}^{2}(x) \phi_{0}(x, y)\right)+\Omega_{\mathrm{d} x}^{2}(x, \infty) \frac{\partial \phi_{0}(x, y)}{\partial y} \quad\left(y \geq y_{\infty}\right) .
\end{aligned}
$$

The odd solution of these equations outside the boundary layer is

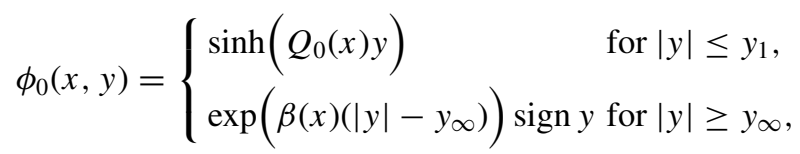

where

$$
\beta(x)=-\sqrt{Q_{0}^{2}(x)-\frac{\Omega_{\mathrm{d} x}^{2}(x, \infty)}{4 S^{2}}}+\frac{i \Omega_{\mathrm{d} x}(x, \infty)}{2 \mathrm{~S}} \approx-Q_{0}(x) .
$$

The solutions (33) allow us to find boundary conditions for Eq. (31) at $y=0$ and $|y|=y_{\infty}$.

It follows from Eq. (28) that we can take $\phi_{0}(x, y)$ as the first partial solution of Eq. (27), and set

$$
f_{01}(x, y)=\phi_{0}(x, y)=\left\{\begin{array}{l}
f_{11}(x, y) \text { for } 0 \leq y \leq y_{0} \\
f_{12}(x, y) \text { for } y_{0} \leq y \leq y_{\infty}
\end{array}\right.
$$

where $f_{11}(x, y)$ and $f_{12}(x, y)$ are partial solutions of Eq. (27) with the boundary conditions

$$
\begin{gathered}
f_{11}(x, 0)=0, \quad \frac{\partial f_{11}(x, 0)}{\partial y}=Q_{0}(x), \quad \frac{\partial^{2} f_{11}(x, 0)}{\partial y^{2}}=0, \quad \frac{\partial^{3} f_{11}(x, 0)}{\partial y^{3}}=Q_{0}^{3}(x), \\
f_{12}\left(x, y_{\infty}\right)=1, \quad \frac{\partial f_{12}\left(x, y_{\infty}\right)}{\partial y}=\beta, \quad \frac{\partial^{2} f_{12}\left(x, y_{\infty}\right)}{\partial y^{2}}=\beta^{2}, \quad \frac{\partial^{3} f_{12}\left(x, y_{\infty}\right)}{\partial y^{3}}=\beta^{3}(x) .
\end{gathered}
$$

For the nonzero roots $g_{1,2}(x, y)$, we can equate in Eq. (27) the coefficients of $\lambda^{3}$ to find the equation for function $\phi_{0}(x, y)$. In view of Eq. (29), this equation is

$$
\frac{\partial \phi_{0}(x, y)}{\partial y}+\frac{2\left(5 g_{1,2}(x, y)-v_{\mathrm{d}}(x, y)\right) \partial g_{1,2}(x, y) / \partial y-\Omega_{\mathrm{d} x}(x, y)}{g_{1,2}(x, y)\left(4 g_{1,2}(x, y)-v_{\mathrm{d}}(x, y)\right)} \phi_{0}(x, y)=0 .
$$

It has to be solved with the boundary conditions

$$
\phi_{0}(x, 0)=0 \quad \text { for } 0 \leq y \leq y_{0}, \quad \phi_{0}\left(x, y_{\infty}\right)=1 \quad \text { for } y \sim y_{\infty}
$$


Such a partial solution is

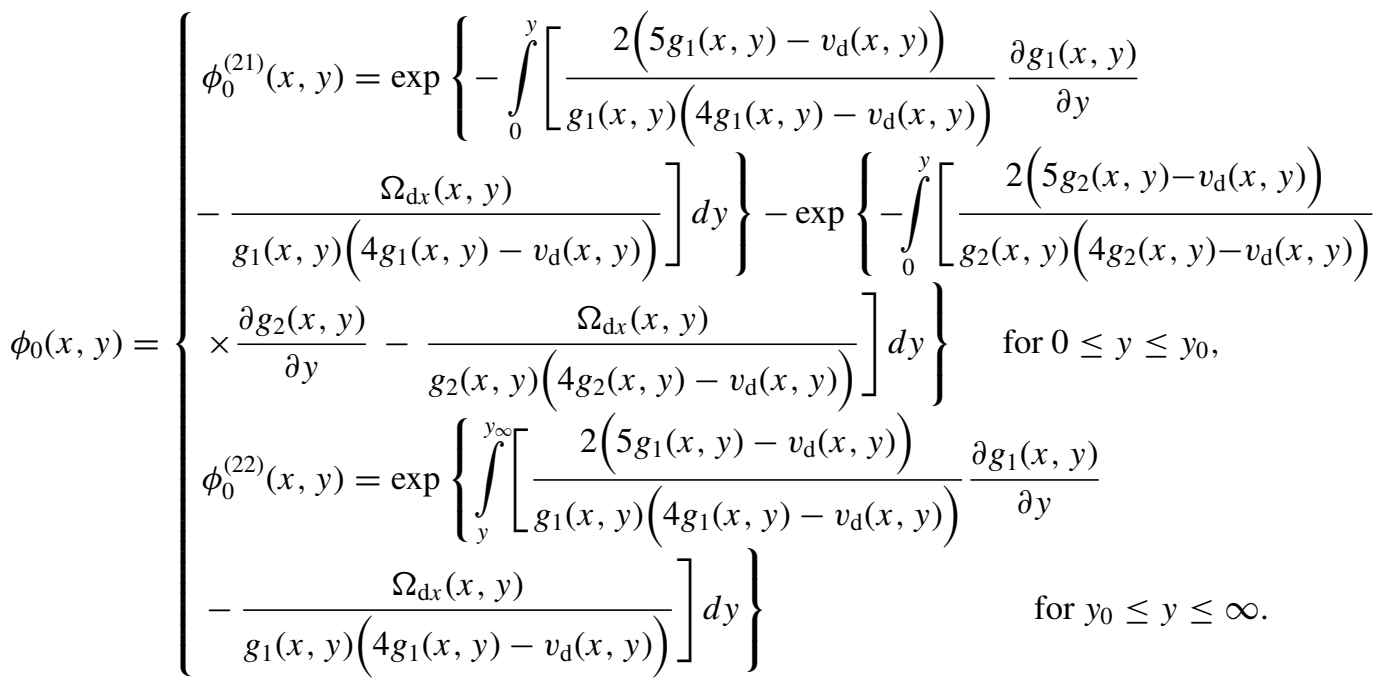

It follows from Eqs. (28) and (40) that the second partial solution of Eq. (27) is

$$
f_{02}(x, y)=\left\{\begin{array}{l}
f_{21}(x, y) \text { for } 0 \leq y \leq y_{0} \\
f_{22}(x, y) \text { for } y_{0} \leq y \leq y_{\infty}
\end{array}\right.
$$

where

$$
\begin{aligned}
& f_{21}(\mathrm{~S}, x, y)=\exp \left\{\int _ { 0 } ^ { y } \left[\sqrt{\operatorname{Re}} g_{1}(x, y)-\frac{2\left(5 g_{1}(x, y)-v_{\mathrm{d}}(x, y)\right)}{g_{1}(x, y)\left(4 g_{1}(x, y)-v_{\mathrm{d}}(x, y)\right)} \frac{\partial g_{1}(x, y)}{\partial y}\right.\right. \\
& \left.\left.+\frac{\Omega_{\mathrm{d} x}(x, y)}{g_{1}(x, y)\left(4 g_{1}(x, y)-v_{\mathrm{d}}(x, y)\right)}\right] d y\right\}-\exp \left\{\int _ { 0 } ^ { y } \left[\sqrt{\operatorname{Re}} g_{2}(x, y)-\frac{2\left(5 g_{2}(x, y)-v_{\mathrm{d}}(x, y)\right)}{g_{2}(x, y)\left(4 g_{2}(x, y)-v_{\mathrm{d}}(x, y)\right)}\right.\right. \\
& \left.\left.\times \frac{\partial g_{2}(x, y)}{\partial y}+\frac{\Omega_{\mathrm{d} x}(x, y)}{g_{2}(x, y)\left(4 g_{2}(x, y)-v_{\mathrm{d}}(x, y)\right)}\right] d y\right\} \quad 0 \leq y \leq y_{0}(x), \\
& f_{22}(\mathrm{~S}, x, y)=\exp \left\{\int _ { y } ^ { y _ { \infty } } \left[-\sqrt{\operatorname{Re}} g_{1}(x, y)+\frac{2\left(5 g_{1}(x, y)-v_{\mathrm{d}}(x, y)\right)}{g_{1}(x, y)\left(4 g_{1}(x, y)-v_{\mathrm{d}}(x, y)\right)}\right.\right. \\
& \left.\left.\times \frac{\partial g_{1}(x, y)}{\partial y}-\frac{\Omega_{\mathrm{d} x}(x, y)}{g_{1}(x, y)\left(4 g_{1}(x, y)-v_{\mathrm{d}}(x, y)\right)}\right] d y\right\} \quad y_{0}(x) \leq y \leq y_{\infty}(x) \text {. }
\end{aligned}
$$

The general solution of Eq. (27) like as Eq. (22) is

$$
f_{0}(\mathrm{~S}, x, y)=\left\{\begin{array}{l}
C_{11}(x) f_{11}(\mathrm{~S}, x, y)+C_{12}(x) f_{12}(\mathrm{~S}, x, y) \text { for } 0 \leq y \leq y_{0}, \\
C_{21}(x) f_{21}(\mathrm{~S}, x, y)+C_{22}(x) f_{22}(\mathrm{~S}, x, y) \text { for } y \geq y_{0},
\end{array}\right.
$$

where $C_{11}(x), C_{12}(x), C_{21}(x)$, and $C_{22}(x)$ are arbitrary functions of $x$. The sewing conditions are the same as Eq. (24).

The complex eigenvalues $Q_{0}(\mathrm{~S}, x)$ should be found from the condition of vanishing the determinant $D\left(Q_{0}(\mathrm{~S}, x)\right)$ of system (24) at the sewing point $y_{0}$, i.e., we must solve the system of two 

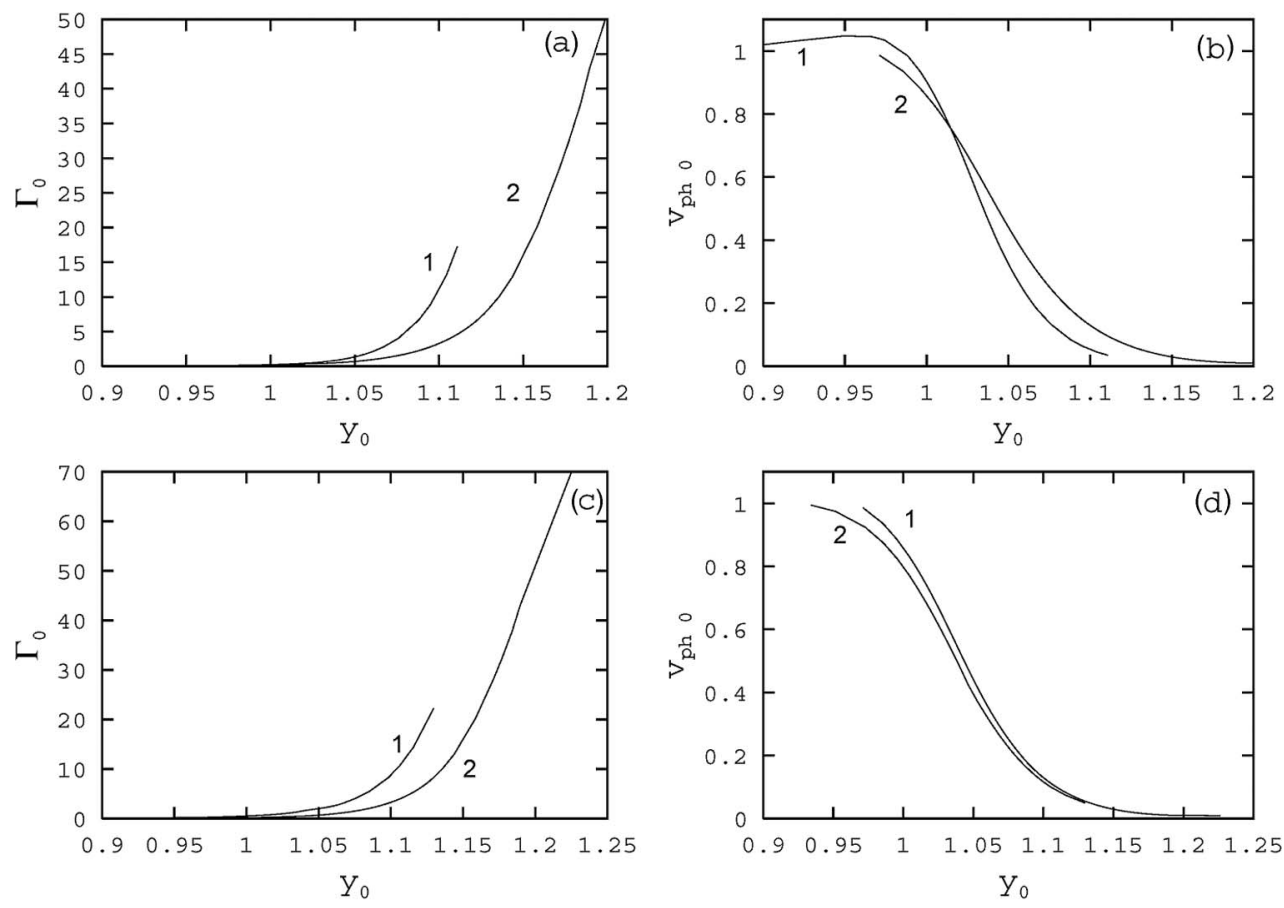

FIG. 3. The dependences $(a, c)$ of the gain factor $\Gamma_{0}=\operatorname{Im}\left(Q_{0}\right)$ and $(b, d)$ the wave phase velocity $v_{\mathrm{ph} 0}=\mathrm{S} / \operatorname{Re}\left(Q_{0}\right)$ on the sewing-point $y_{0}$ for (a) $\mathrm{S}=1$ and two values of $x(x=4$ (1) and $x=8$ (2)); and for (b) $x=8$ and two values of $S(S=1$ (1) and $S=4(2)$ ).

equations

$$
\operatorname{Re}\left(D\left(Q_{0}(\mathrm{~S}, x)\right)\right)=0, \quad \operatorname{Im}\left(D\left(Q_{0}(\mathrm{~S}, x)\right)\right)=0
$$

with respect to two unknowns $\operatorname{Re}\left(Q_{0}(\mathrm{~S}, x)\right)$ and $\operatorname{Im}\left(Q_{0}(\mathrm{~S}, x)\right)$.

Different methods for numerical solution of such systems are described, for example, in Refs. 51 and 52. They all are based on the supposition that there exists a functional monotonically changing with the changes in $\left|D\left(Q_{0}(\mathrm{~S}, x)\right)\right|$. In our case, however, such a functional does not exist. We have therefore used a trial-and-error method. Our calculations have shown that the roots of Eqs. (45) depend essentially from the sewing point $y_{0}$.

Examples of the dependences of the gain factor $\Gamma_{0}=\operatorname{Im}\left(Q_{0}\right)(a, c)$ and the wave phase velocity $v_{\mathrm{ph} 0}=\mathrm{S} / \operatorname{Re}\left(Q_{0}\right)(b),(d)$ on the sewing-point $y_{0}$ are shown in Fig. 3 for $\mathrm{S}=1$ and two values of $x$ $(x=4(1)$ and $x=8(2))$; and for $x=8$ and two values of $S(S=1$ (1) and $S=4$ (2)).

\section{DISCUSSION}

The fact that the eigenvalues and eigenfunctions, corresponding to the same frequencies, are different for different sewing points means that an infinite number of waves of the same frequency but with different wavelengths may coexist. Because of the instability, the amplitudes of these waves become random. Consequently, hydrodynamic waves in inhomogeneous media do not have a unique dispersion law, i.e., different phase velocities may correspond to the same frequencies. Seemingly, in such media turbulence arises in just this way.

We see that the phase velocity takes on all values from 1 , when the sewing-point approaches the inner boundary of the boundary layer, to 0 , when the sewing-point approaches its external boundary. Correspondingly, the gain factor changes from zero to a finite limiting value that is sufficiently 

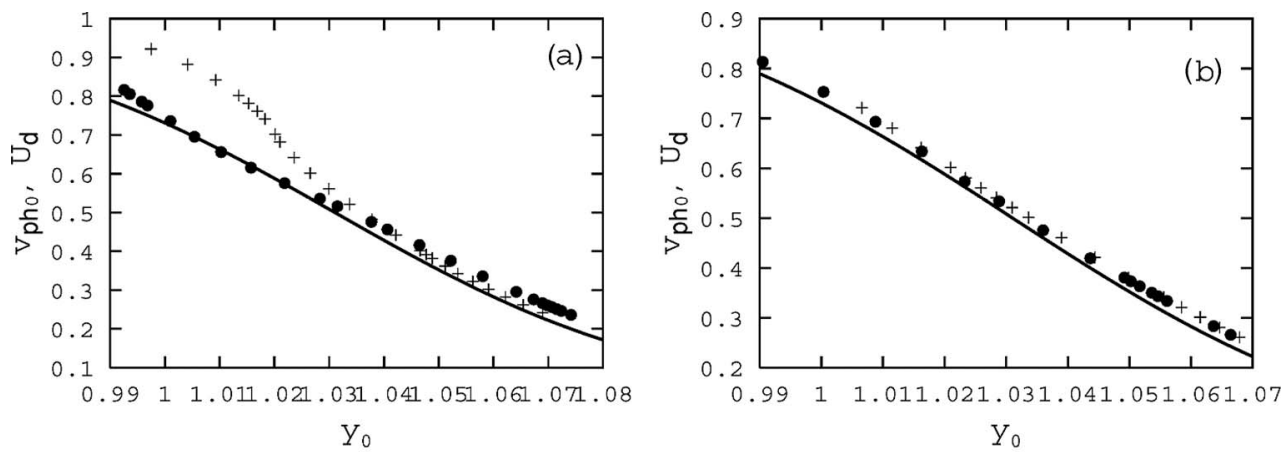

$\mathrm{Y}_{0}$

(c)

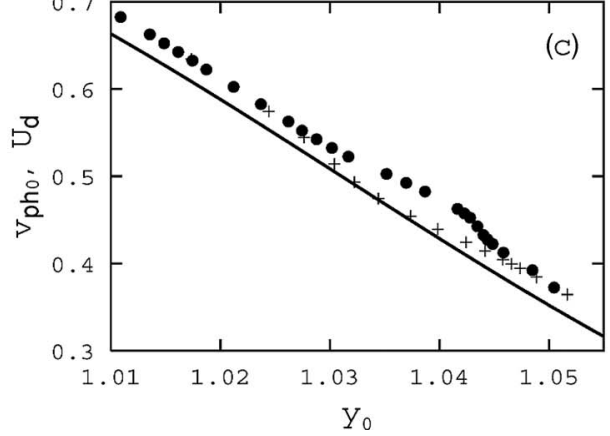

FIG. 4. The dependences of the phase velocities on sewing-point $y_{0}$ for $x=8$ : (a) $\mathrm{S}=1$ crosses and $\mathrm{S}=2$ circles; (b) $\mathrm{S}=3$ crosses and $\mathrm{S}=4$ circles; (c) $\mathrm{S}=4.5$ crosses and $\mathrm{S}=5$ circles; the dependence of the dynamical longitudinal flow velocity is shown in $\mathrm{a}, \mathrm{b}$, and $\mathrm{c}$ by heavy line.
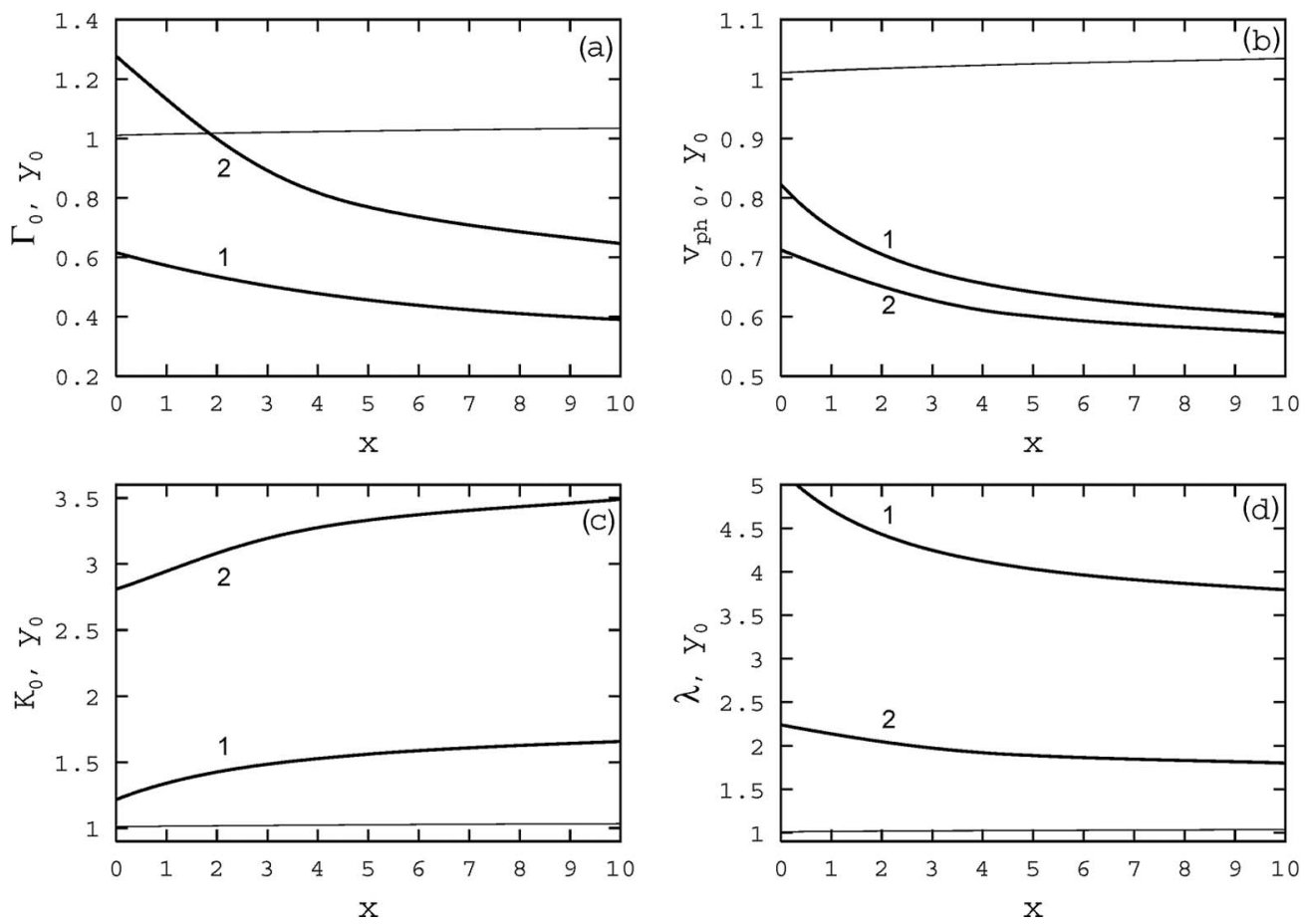

FIG. 5. The dependences of (a) the gain factor $\Gamma_{0}$, (b) the wave phase velocity $v_{\mathrm{ph} 0}$, (c) the wave number $K_{0}$ and (d) the wavelength $\lambda$ on the distance from the nozzle $x$ for $S=2$. 
large. It should be noted that negative values of the phase velocity signify that the corresponding hydrodynamic wave propagates toward the jet flow and damps. For comparatively large values of $y_{0}, x=8$, we have found two eigenvalues for each value of $y_{0}$.

It is very interesting that, as can be seen from Fig. 4, for any $x$ and $y_{0}$ the phase velocity of the waves is close to the dynamical constituent of the longitudinal flow velocity $U_{\mathrm{d}}\left(x, y_{0}\right)$ (see Fig. (3)). This feature can be explained by the fact that, as shown in Ref. 53 in the linear approximation hydrodynamic waves in jets are transverse and propagate downstream with the flow velocity. The ratio $v_{\mathrm{ph}}\left(y_{0}\right) / U_{\mathrm{d}}\left(y_{0}\right)$ is sensibly independent of $\mathrm{S}$ and at any sewing-point lies close to unity. The well known experimental fact that, when interacting with an obstacle, hydrodynamic waves transform partially into acoustic waves ${ }^{17}$ may be easily accounted for by the transverse nature of hydrodynamic waves.

Figure 5 illustrates the dependences of the gain factor $\Gamma_{0}$, phase velocity $v_{\text {ph } 0}$, wave number $K_{0}$, and wavelength $\lambda$ on the distance $x$ from the nozzle at fixed values of the Strouhal number. The corresponding values of the sewing point coordinate $y_{0}$ are shown by thin lines. The cause of the decrease in wavelength as the distance increases lies in the decrease in phase velocity with an increase of the sewing point coordinate (see Fig. 3).

It should be noted that all dependences of the gain factor $\Gamma_{0}$ on the Strouhal number $S$ are of a resonant character. For a certain value of $S$ these dependences take on a maximal value depending on the distance from the nozzle. This may be treated as indicating that a jet may be represented as a set of resonators, as discussed in Ref. 17.

\section{CONCLUSIONS}

There are several significant conclusions to be drawn. First, splitting the initial equations into regular and random constituents is a useful approach that does not result in problems of closure. Secondly, the transition to turbulence occurs via the generation of an infinite number of waves: there is a continuum of frequencies and, for each frequency, a continuum of phase velocities. Correspondingly, there is an infinite number of wavelengths. It follows that there is no unique dispersion law and, because of perturbations (however small they may be), a regular temporal spectrum does not exist even in cases where the spatial spectrum is regular. Thirdly, for all values of frequency, and of other parameters, the wave phase velocity lies close to the flow velocity, which may be accounted for by the fact that hydrodynamic waves propagating downstream with the flow velocity, and acoustic waves, are solutions of the same hydrodynamic equations. ${ }^{53}$

The importance of these results relates not only to the fundamental understanding of turbulent processes in jets, but also bears on many practical applications including those mentioned as examples in Sec. I. They are also of more general applicability, to all cases where turbulence arises in an inhomogeneous medium, e.g., the atmosphere.

${ }^{1}$ D. J. Tritton, Physical Fluid Dynamics, 2nd ed. (Clarendon, Oxford, 1988).

${ }^{2}$ P. A. Davidson, Turbulence: An Introduction for Scientists and Engineers (Oxford University Press, Oxford, 2004).

${ }^{3}$ V. S. Tsoi, "Transverse electron focusing as a way of studying phonon kinetics. Turbulence of phonon flow," Cent. Eur. J. Phys. 1, 72 (2003).

${ }^{4}$ U. Bortolozzo, J. Laurie, S. Nazarenko, and S. Residori, "Optical wave turbulence and the condensation of light," J. Opt. Soc. Am. B 26, 2280 (2009).

${ }^{5}$ A. Boudaoud, O. Cadot, B. Odille, and C. Touze, "Observation of wave turbulence in vibrating plates," Phys. Rev. Lett. 100, 234504 (2008).

${ }^{6}$ N. Mordant, "Are there waves in elastic wave turbulence?," Phys. Rev. Lett. 100, 234505 (2008).

${ }^{7}$ P. Cobelli, P. Petitjeans, A. Maurel, V. Pagneux, and N. Mordant, "Space-time resolved wave turbulence in a vibrating plate," Phys. Rev. Lett. 103, 204301 (2009).

${ }^{8}$ F. Boyer and E. Falcon, "Wave turbulence on the surface of a ferrofluid in a magnetic field," Phys. Rev. Lett. 101, 244502 (2008).

${ }^{9}$ E. Falcon, "Laboratory experiments on wave turbulence," Discrete Contin. Dyn. Syst., Ser. B 13, 819 (2010).

${ }^{10}$ S. N. Gurbatov, V. V. Kurin, L. M. Kustov, and N. V. Pronchatov-Rubtsov, "Physical modeling of nonlinear sound wave propagation in oceanic waveguides of variable depth," Acoust. Phys. 51, 152 (2005).

${ }^{11}$ G. S. Bisnovatyi-Kogan and S. A. Silich, "Shock-wave propagation in the nonuniform interstellar medium," Rev. Mod. Phys. 67, 661 (1995).

${ }^{12}$ M. Ryutova and T. Tarbell, "MHD shocks and the origin of the solar transition region," Phys. Rev. Lett. 90, 191101 (2003). 
${ }^{13}$ G. V. Kolmakov, A. A. Levchenko, M. Y. Brazhnikov, L. P. Mezhov-Deglin, A. N. Silchenko, and P. V. E. McClintock, "Quasi-adiabatic decay of capillary turbulence on the charged surface of liquid hydrogen," Phys. Rev. Lett. 93, 074501 (2004).

${ }^{14}$ G. V. Kolmakov, V. B. Efimov, A. N. Ganshin, P. V. E. McClintock, and L. P. Mezhov-Deglin, "Formation of a direct Kolmogorov-like cascade of second sound waves in He II," Phys. Rev. Lett. 97, 155301 (2006).

${ }^{15}$ A. N. Ganshin, V. B. Efimov, G. V. Kolmakov, L. P. Mezhov-Deglin, and P. V. E. McClintock, "Observation of an inverse energy cascade in developed acoustic turbulence in superfluid helium," Phys. Rev. Lett. 101, 065303 (2008).

${ }^{16}$ V. E. Zakharov, V. S. L’vov, and G. Falkovich, Kolmogorov Spectra of Turbulence I (Springer, Berlin, 1992).

${ }^{17}$ P. S. Landa and P. V. E. McClintock, "Development of turbulence in subsonic submerged jets," Phys. Rep. 397, 1 (2004).

${ }^{18}$ P. S. Landa, Nonlinear Oscillations and Waves (URSS, Moscow, 2010).

${ }^{19}$ P. S. Landa, Nonlinear Oscillations and Waves in Dynamical Systems (Kluwer, Dordrecht, 1996).

${ }^{20}$ L. D. Landau and E. M. Lifshitz, Fluid Mechanics (Butterworth and Heinemann, Oxford, 1987).

${ }^{21}$ P. S. Landa, "Turbulence in nonclosed fluid flows as a noise-induced phase transition," Europhys. Lett. 36, 401 (1996).

${ }^{22}$ P. S. Landa, "Turbulence and coherent structures in subsonic jets. Control of the turbulence," in Proceedings of ICNBC, (ICNBC, Lodz-Dobieszkov, 1996), pp. 40-45.

${ }^{23}$ P. S. Landa, "Onset of turbulence in open liquid flows as a nonequilibrium noise-induced $\backslash$ ond-order phase transition," Tech. Phys. 43, 27 (1998).

${ }^{24}$ P. S. Landa and A. S. Ginevsky, "Control of Turbulence in Jets by Acoustic Means," in Proceedings of International Conference on Physics and Control (IEEE, St. Petersburg, 2003), pp. 31-40.

${ }^{25}$ A. S. Ginevsky, P. S. Landa, and A. A. Zaikin, "Self-excitation of impinging jets with regard to acoustic feedback," in Proceedings of Third International Congress on Air- and Structure-borne Sound and Vibration (Montreal, 1994), pp. 1191-1198.

${ }^{26}$ P. S. Landa, Self-oscillations in Spatially Extended Systems (URSS, Moscow, 2009).

${ }^{27}$ D. G. Crighton and M. Gaster, "Stability of slowly diverging jet flow," J. Fluid Mech. 77, 397 (1976).

${ }^{28}$ P. Plaschko, "Helical instabilities of slowly diverging jets," J. Fluid Mech. 92, 209 (1979).

${ }^{29}$ P. Plaschko, "Axial coherence functions of circular turbulent jets based on an inviscid calculation of damped modes," Phys. Fluids 26, 2368 (1983).

${ }^{30}$ A. Michalke, "Survey on jet instability theory," Prog. Aerosp. Sci. 21, 159 (1984).

${ }^{31}$ M. Gaster, "On the growth of waves in boundary layers: A non-parallel correction," J. Fluid Mech. 424, 367 (2000).

${ }^{32}$ J. C. R. Hunt and D. J. Carruthers, "Rapid distortion theory and the 'problems' of turbulence," J. Fluid Mech. 212, 497 (1990).

${ }^{33}$ P. W. Terry, "Suppression of turbulence and transport by sheared flow," Rev. Mod. Phys. 72, 109 (2000).

${ }^{34}$ A. S. Monin and A. M. Yaglom, Statistical Fluid Mechanics: Mechanics of Turbulence (Dover, Mineola, NY, 2007), Vol. 1.

${ }^{35}$ B. G. Galerkin, Collected Works (Izd-vo AN SSSR, Moscow, 1952), Vol. 1.

${ }^{36}$ A. S. Ginevsky, The Theory of Turbulent Jets and Wakes (Mashinovedenie, Moscow, 1969) (in Russian).

${ }^{37}$ S. M. Belotserkovsky and A. S. Ginevsky, Simulation of Turbulent Jets and Wakes by Discrete Vortex Technique (Nauka, Moscow, 1995).

${ }^{38}$ H. Schlichting, Grenzschicht-Theorie (C. Braun, Karlsruhe, 1965).

${ }^{39}$ G. Wentzel, "Eine verallgemeinerung der quantenbedingungen für die zwecke der wellenmechanik," Z. Phys. 38, 518 (1926).

${ }^{40}$ H. A. Kramers, “Wellenmechanik und halbzählige quantisierung,” Z. Phys. 39, 828 (1926).

${ }^{41}$ L. Brillouin, "La mécanique ondulatoire de Schrödinger une méthode générale de resolution par approximations suscessives," Compt Rendus de l'Academie des Sciences 183, 24 (1926).

${ }^{42}$ H. Poincaré, Sur les Courbes dF́inies par les Équations Différentielles (Gauthier-Villars, Paris, 1886).

${ }^{43}$ G. D. Birkhoff, "On the asymptotic character of the solutions of certain linear differential equations containing a parameter," Trans. Am. Math. Soc. 9, 219 (1908).

${ }^{44}$ L. Rayleigh, "On the propagation of waves through a stratified medium, with special reference to the question of reflection," Proc. R. Soc. London, Ser. A 86, 207 (1912).

45 J. D. Tamarkin, On Some General Problems in the Theory of Ordinary Linear Differential Equations and on the Expansion in Series of Arbitrary Functions (Petrograd, 1917) (in Russian).

${ }^{46}$ J. D. Tamarkin, "Some general problems of the theory of ordinary linear differential equations and expansion of an arbitrary function in series of fundamental functions," Math. Zeit. 27, 1 (1927).

47 J. Heading, An Introduction to Phase-Integral Methods (Wiley, New York, 1962).

${ }^{48}$ N. Fröman and P. O. Fröman, JWKB Approximation (North-Holland, Amsterdam, 1965).

${ }^{49}$ M. V. Fedoryuk, Asymptotic Methods for Linear Ordinary Differential Equations (Nauka, Moscow, 1977) (in Russian).

${ }^{50}$ V. P. Maslov and M. V. Fedoryuk, Quasiclassical Approximation for Quantum-Mechanical Equations (Nauka, Moscow, 1975).

${ }^{51}$ G. A. Korn and T. M. Korn, Mathematical Handbook (McGraw-Hill, New York, 1968).

${ }^{52}$ Y. I. Neimark, N. Y. Kogan, and V. P. Savel'ev, Dynamical Models of Control Theory (Nauka, Moscow, 1985).

${ }^{53}$ P. S. Landa, D. I. Trubetskov, and V. A. Gusev, "Delusions versus reality in some physics problems: theory and experiment," Physics-Uspekhi 52, 235 (2009).

${ }^{54}$ H. Jeffreys, "On certain approximate solutions of linear differential equations of the second order," Proc. London Math. Soc. 23, 428 (1924).

${ }^{55}$ A similar method was suggested somewhat earlier, by Jeffreys (Ref. 54). So in the literature the method is often named JWKB. 